Article

\title{
Ensemble Mode and Singular Value Based Road Texture-Skid Study
}

\author{
Tuo Fang ${ }^{1} \mathbb{D}$, Runhua Guo ${ }^{2, * \mathbb{C}}$ and Youqiang $\mathrm{Si}^{3}$ \\ 1 School of Civil and Environmental Engineering, University of New South Wales (UNSW), \\ Sydney 2052, Australia; fangtuo1202@gmail.com \\ 2 Department of Civil Engineering, Tsinghua University, Beijing 100084, China \\ 3 Shandong Hi-Speed Linyi Operation Management Center, Zaozhuang 277000, China; 18801188337@163.com \\ * Correspondence: guorh@tsinghua.edu.cn
}

check for

updates

Citation: Fang, T.; Guo, R.; Si, Y. Ensemble Mode and Singular Value Based Road Texture-Skid Study. Coatings 2021, 11, 947. https:// doi.org/10.3390/ coatings11080947

Academic Editor: Malal Kane

Received: 28 May 2021

Accepted: 4 August 2021

Published: 8 August 2021

Publisher's Note: MDPI stays neutral with regard to jurisdictional claims in published maps and institutional affiliations.

Copyright: (c) 2021 by the authors. Licensee MDPI, Basel, Switzerland. This article is an open access article distributed under the terms and conditions of the Creative Commons Attribution (CC BY) license (https:// creativecommons.org/licenses/by/ $4.0 /)$.

\begin{abstract}
Traffic accidents occur frequently every year. Skid-resistance performance is an important indicator in evaluating road safety. In this paper, the road surface texture information is obtained by a line laser scanner. The original two-dimensional (2D) signal is decomposed into eight intrinsic mode functions by complementary ensemble empirical mode decomposition (CEEMD). Eight intrinsic mode functions compose an $800 \times 8$ data matrix. The matrix is decomposed by singular value decomposition (SVD), and singular value vectors are obtained. The singular values are summed and linearly fitted to the friction value measured by the British pendulum friction meter. The study finds that the singular value sum of one to five has a good linear correlation with the friction value with the goodness of fit greater than 0.8. At the same time, contour arithmetic mean deviation $R_{a}$ is applied for verification. The results show that the singular value sum has a strong correlation with $\mathrm{R}_{\mathrm{a}}$. When the number of Intrinsic Mode Function (IMF) decomposition is greater than eight, there is a good correlation between the number of IMF and the friction value from IMF2 to IMF6. When the number of IMF decomposition is less than eight, the singular value sum numbers with $\mathrm{R}^{2}$ more than 0.8 gradually decrease. It is suggested that the road surface friction performance can be estimated by solving the singular value of the $2 \mathrm{D}$ signal. It provides new technical support for the detection and evaluation of anti-skid performance.
\end{abstract}

Keywords: pavement texture; CEEMD singular value decomposition; skid-resistance performance

\section{Introduction}

Traffic accidents happen frequently, and subsequently, road safety has become an urgent problem in the world. Skid-resistance is an important indicator in evaluating road safety performance. Although there are different factors involved in driving that affect safety, such as vehicle performance and climate conditions, effective skid-resistance of pavement is the basic condition and essential guarantee for safe driving. In traffic accidents that are significantly affected by braking, the skid-resistance of the road surface could, to a certain extent, reduce the severity of the accident, or even avoid the occurrence of the traffic accident altogether. According to the British survey data [1], the proportion of accidents caused by smooth road surface is $24 \%$ of the total number of accidents in the year, while the sample survey in Japan indicates that the proportion of accidents caused by a smooth road surface is about $25 \%$ of the total number of accidents in the year. Therefore, skid-resistance detection of pavement has become one of the most important tasks to ensure road safety. It would be useful to provide a methodology for averaging the value of the slip resistance indicator for the purpose of taking it into account in the investigation of road accidents.

The measurement for skid-resistance is mainly concerned with contact method. The British Road Transport Institute invented the pendulum friction coefficient tester, or the British Pendulum Tester (BPT). It is one of the earliest established methods and standards for evaluating the skid-resistance performance of the road. Fwa et al. [2] used the Abaqus 
simulated pendulum friction tester to find that the numerical simulation can better simulate the length of the friction block slip and the value of the pendulum during the actual measurement process, which is consistent with the actual test results.

In recent years, non-contact detection technology has gradually developed. For example, laser technology and camera photography technology have been applied to the road surface characteristic measurement. Zahouani et al. [3] applied the laser tracking focusing method to test the texture of the asphalt pavement by extracting the microscopic texture of the pavement. However, this method was only used for the measurement of 2D contours. Through the principle of laser triangulation, the surface topography can be reconstructed for rut depth, flatness, and macrotexture [4]. In 2016, Fang et al. [5] used the matrix laboratory algorithm to process a single aggregate image, and conducted a Fourier analysis. The results of the matrix laboratory algorithm based on the Fourier function were accurate, and could be used to characterize the surface morphology of aggregates.

At present, the commonly used evaluation indexes of road skid-resistance performance are mainly based on the friction coefficient index (e.g., BPN, lateral force coefficient), the texture index fractal dimension, Mean Texture Depth (MTD), Mean Profile Depth (MPD), etc. So far, a large number of scholars and engineers have investigated predictive models by examining the correlation between different texture indexes and road friction coefficients [6,7]. Chou [8] proposed a new laser-based evaluation index-MDE (mean difference of elevation) — based on the existing MPD and MTD. The MDE is the sum of the fixed height differences of every two adjacent points in the measurement length divided by the number of points on the measured length. Meegoda [9] studied the relationship between the friction coefficient and the MPD value. The study found that the distribution of MPD values had both positive and negative correlations with the friction coefficient. On this basis, the relationships between texture, structure, depth, tire contact area, water film thickness, and friction coefficient were studied. Zhou et al. [10] studied the fractal characteristics of pavement texture under different polishing conditions by fractal theory, and proposed the concept of the cutoff wavelength as an index for evaluating ordinary polishing and differential polishing. The horizontal cutoff wavelength indicates the average microprotrusion size. The cutoff wavelength indicates the average height of the aggregate. A three-dimensional (3D) laser scanner was used to extract different polished texture information. Yang et al. [11] used the multi-function road condition rapid detection system (CiCS) to collect the pavement texture depth information, to calculate the road surface wear rate, to compare and analyze the MTD and MPD values of the road wear surface, and to establish the average of road wear and road texture. The evaluation model between the depth was constructed. The model was applied to the texture evaluation index standard. Rado and Cho et al. [12,13] proposed to process the 2D texture signal by Hilbert-Huang transform (HHT). They obtained the instantaneous envelope and instantaneous amplitude of the joint intrinsic mode function BIMF by Hilbert-Huang (HHT) transform to extract the peak envelope. A good linear correlation was found between the mean value and the friction coefficient.

It is noted that the evaluation of pavement texture by laser scanning technology is mainly focused on MTD and MPD. Due to the limitations in the accuracy of the texture detection device, it can only collect macrotexture at traffic speed in the field. Microtexture collection can only be done in the laboratory. Even within the macrotexture data, the quality is poor due to vehicle vibration and speed. In this study, a multi-function inspection vehicle texture sensor or module is used. The vehicle adopts a non-inertial system, which is not affected by the driving speed and the vibration of the vehicle. The 3D texture information can be quickly collected at a high speed. The accuracy of the texture module is up to $0.01 \mathrm{~mm}$. The wavelength can cover all of the macrotexture and some of the microtexture. At the same time, this paper puts forward a new index to evaluate the anti-slip performance by processing the collected data in an innovative manner. Compared with other evaluation indexes, this index has a better correlation with anti-slip performance. 
This study aims to improve the modeling capacity between texture and skid. A different novel modeling approach was employed to analyze the texture data in order to correlate with roadway skid.

\section{Data Collection Technology}

To better study the friction performance of the road surface, a laser line-scanning texture device attached on a multi-function inspection vehicle was employed in this research (Figure 1). The skid-resistance of the surface was measured by the British Pendulum Tester. The advantage of this device/module is that it is not affected by the driving speed and the vehicle vibration. The elevation resolution reaches $10 \mu \mathrm{m}$. When the line laser is installed along the travel direction above the wheel paths, the 2D texture on the center line of the wheel paths can be measured while traveling. Alternatively, while the line laser is installed perpendicular to the travel direction, the 3D texture can be obtained by combining multiple $2 \mathrm{D}$ data.

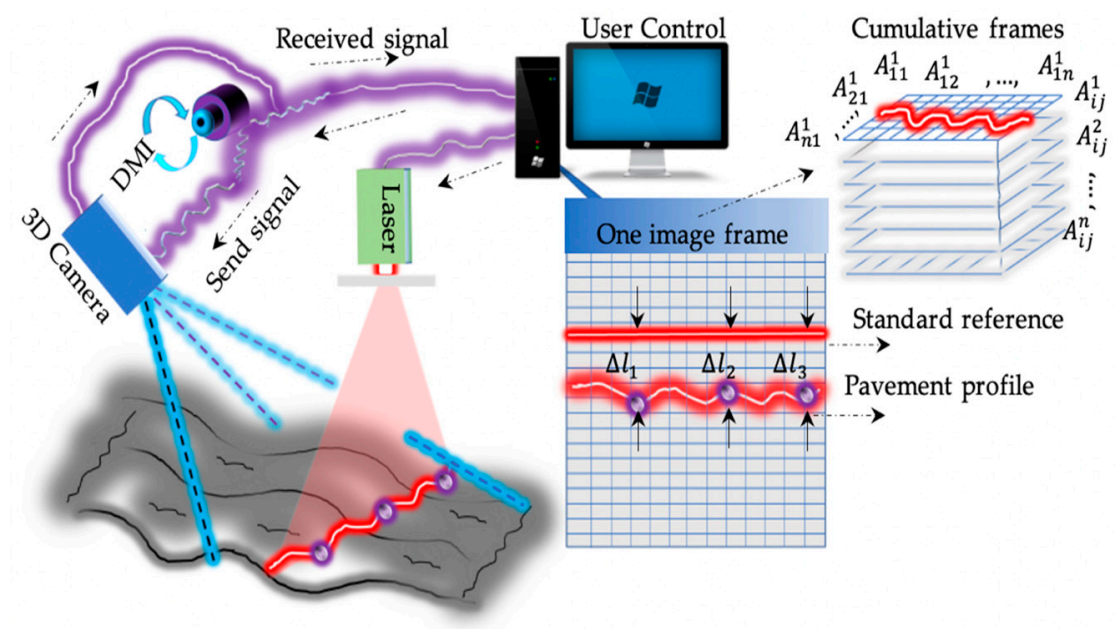

Figure 1. Schematic diagram of 3D profilometer.

In this test, a total of 8 sets of $300 \mathrm{~mm} \times 300 \mathrm{~mm}$ testing areas were selected on the Hot Mix Asphalt (HMA) surface. The 3D texture of the testing surfaces was collected at a uniform speed using the laser line. Figure 2 shows that the texture information of the testing area was collected by the sensor. The 2D signal on the top of Figure 2 is one line of elevation information of the surface. The bottom of Figure 2 displays a 3D texture surface image generated by aggregating 512 2D lines from the laser scanning.

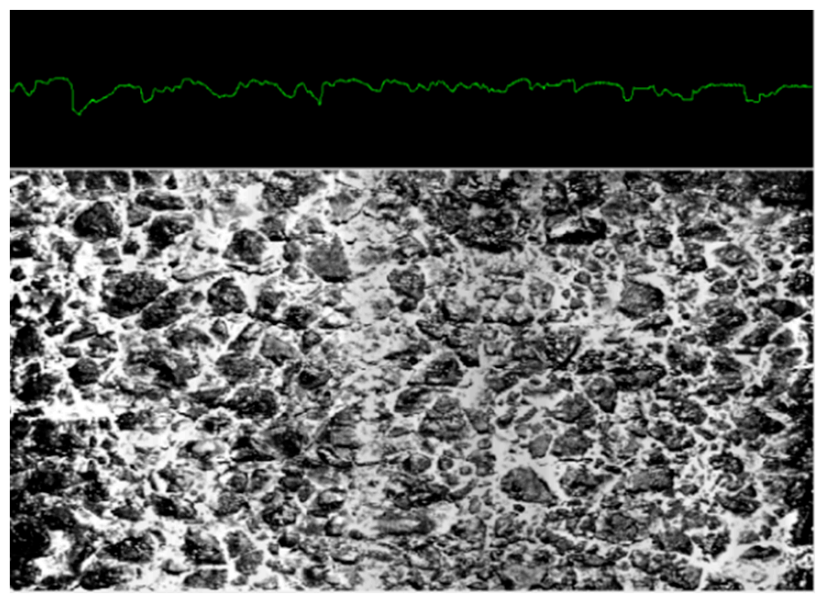

Figure 2. Texture profile and image on HMA surface $(100 \mathrm{~mm} \times 100 \mathrm{~mm})$. 
To be similar to the testing area of the British Pendulum Tester, the center area of the testing area was taken as $100 \mathrm{~mm} \times 100 \mathrm{~mm}$ (Figure 3), and the corresponding data collected by the laser sensor was $800 \times 300$ data matrix. The data of five pendulum measurements and their average was recorded. The pendulum size was corrected according to the change of temperature.

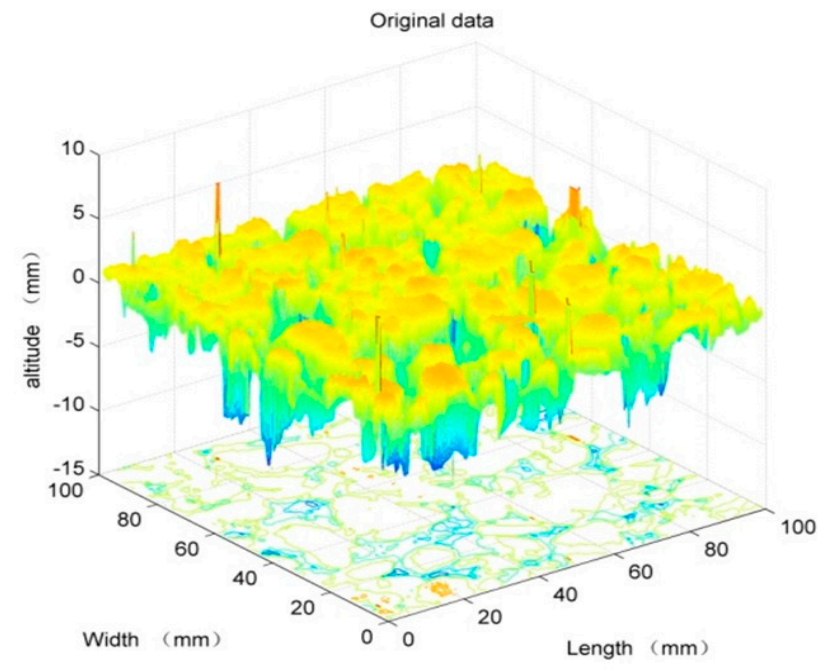

Figure 3. 3D texture reconstruction.

\section{Principle in Data Processing}

\subsection{CEEMD Principle}

The 3D texture data collected by the laser scanner contains a wealth of surface elevation information. They are composed of 300 columns of 2D nonlinear, non-stationary signals, including various noise interferences and multi-frequency mixed signals. For this type of data processing, Huang et al. [14,15] proposed an empirical mode decomposition (EMD) algorithm to decompose the original signal $s(t)$ into more intrinsic mode functions IMF and a remainder. EMD can decompose complex signals into single mode signals with different frequencies. The signals of different frequency segments have different physical meanings. By analyzing the IMF's physical meanings, we can reveal the texture characteristics. For example, for any time series signal $s(t)$, it can be decomposed into $n$ intrinsic mode functions IMF and a remainder.

$$
\mathrm{s}(\mathrm{t})=\sum_{\mathrm{i}=1}^{\mathrm{n}} \operatorname{IMF}_{\mathrm{i}}(\mathrm{t})+\mathrm{r}_{\mathrm{n}}(\mathrm{t})
$$

where $\operatorname{IMF}_{i}(t)$ is the intrinsic mode function and $r_{n}(t)$ is the remainder.

Due to the severe modal aliasing of the EMD algorithm, the original signal reconstruction is poor. Therefore, WU et al. [16] proposed an improved EMD algorithm named ensemble empirical mode decomposition (EEMD). EEMD eliminates the modal aliasing of EMD by continuously adding Gaussian white noise to the original signal.

$$
\mathrm{IMF}_{\mathrm{i}}=\frac{1}{\mathrm{~N}} \sum_{\mathrm{i}=1}^{\mathrm{N}} \mathrm{C}_{\mathrm{ij}}(\mathrm{t})
$$

where $\mathrm{N}$ is the number of added white noise, and $C_{i j}(t)$ represents the $i$-th IMF after the $\mathrm{j}$-th white noise processing is added. Due to the addition of white noise, EEMD cannot be completely eliminated during signal processing, which affects the purity of the signal.

Furthermore, complementary ensemble empirical mode decomposition (CEEMD) is an optimization based on EEMD, which can effectively overcome modal aliasing and white noise interference and accurately reconstruct signals [17,18]. By adding $\mathrm{N}$ positive and negative auxiliary white noise to the original signal, $2 \mathrm{~N}$ signals are obtained and EMD decomposition is performed for each signal. Finally, the calculation result is combined. The 
CEEMD process is the same as EEMD, except that more noise is added after adding a noise, and the IMFs are decomposed according to EMD.

\subsection{Singular Value Decomposition Principle}

The eigenvalue decomposition commonly can only be applied to square matrices. In reality, most matrices are not square matrices, and singular value decomposition can be applied to any matrix decomposition method. Singular value decomposition is the main step of principal component analysis. Singular value decomposition can reduce the complex big data matrix to a low-dimensional data matrix by extracting principal components. Suppose $\mathrm{C}$ is an $\mathrm{m} \times \mathrm{n}$ order matrix,

$$
\mathrm{C}=\mathrm{U} \sum \mathrm{V}^{\mathrm{T}}
$$

where $\mathrm{U}$ is an $\mathrm{m} \times \mathrm{m}$-order matrix, $\Sigma$ is a semi-positive $\mathrm{m} \times \mathrm{n}$-order diagonal matrix, and $\mathrm{V}^{\mathrm{T}}$ is a transposed matrix of $\mathrm{V}$, which is an $\mathrm{n} \times \mathrm{n}$-order matrix. This decomposition $\mathrm{C}$ is called the singular value decomposition. The element $\delta_{i}$ on the diagonal is the singular value. The singular values are usually arranged in descending order, e.g., $\delta_{1}>\delta_{2}>\delta_{3}>\ldots$ $>\delta_{\mathrm{i}}(1<\mathrm{i} \leq \min (\mathrm{m}, \mathrm{n}))$.

\section{Data Analysis and Results}

The 2D original signal is subjected to CEEMD decomposition, and the number of IMFs is set to eight. In (Figure 4), nine IMFs and one residual Rs10 are obtained after decomposing, wherein IMF1 is the original signal. IMF2 to IMF9 are single mode decomposition functions of the original signal. They are arranged according to the frequency from large to small. The high frequency part contains a portion of the microtexture and macrotexture information. IMF6 and beyond are low frequency signals, representing mega-texture information. The residual Rs10 is the degree of signal change and the degree of stability of signal decomposition. The IMF1 and the Rs10 are removed, and the $800 \times 8$ data matrix is composed of IMF2-9. Then, the matrix is subjected to singular value decomposition by Equation (3) to Equation (5) to obtain a singular value matrix $\sum_{800 \times 8}$. A non-zero singular value vector of each column is then extracted. CEEMD decomposition and singular value decomposition are performed on 300 columns of original data, respectively. The singular values of each column in 300 columns are summed in Equation (4), where $\sigma_{i j}$ represents the $i$-th column row singular value of the 300 columns of the original data; $\sigma_{i}$ represents the sum of the singular values of the ith row of 300 columns of data.

$$
\sum_{800 \times 8}=\left[\begin{array}{cccccccc}
\sigma_{1 j} & 0 & 0 & 0 & 0 & 0 & 0 & 0 \\
0 & \sigma_{2 j} & 0 & 0 & 0 & 0 & 0 & 0 \\
0 & 0 & \sigma_{3 j} & 0 & 0 & 0 & 0 & 0 \\
0 & 0 & 0 & \sigma_{4 j} & 0 & 0 & 0 & 0 \\
0 & 0 & 0 & 0 & \sigma_{5 j} & 0 & 0 & 0 \\
0 & 0 & 0 & 0 & 0 & \sigma_{6 j} & 0 & 0 \\
0 & 0 & 0 & 0 & 0 & 0 & \sigma_{7 j} & 0 \\
0 & 0 & 0 & 0 & 0 & 0 & 0 & \sigma_{8 j} \\
\vdots & \vdots & \vdots & \vdots & \vdots & \vdots & \vdots & \vdots \\
0 & 0 & 0 & 0 & 0 & 0 & 0 & 0
\end{array}\right]
$$



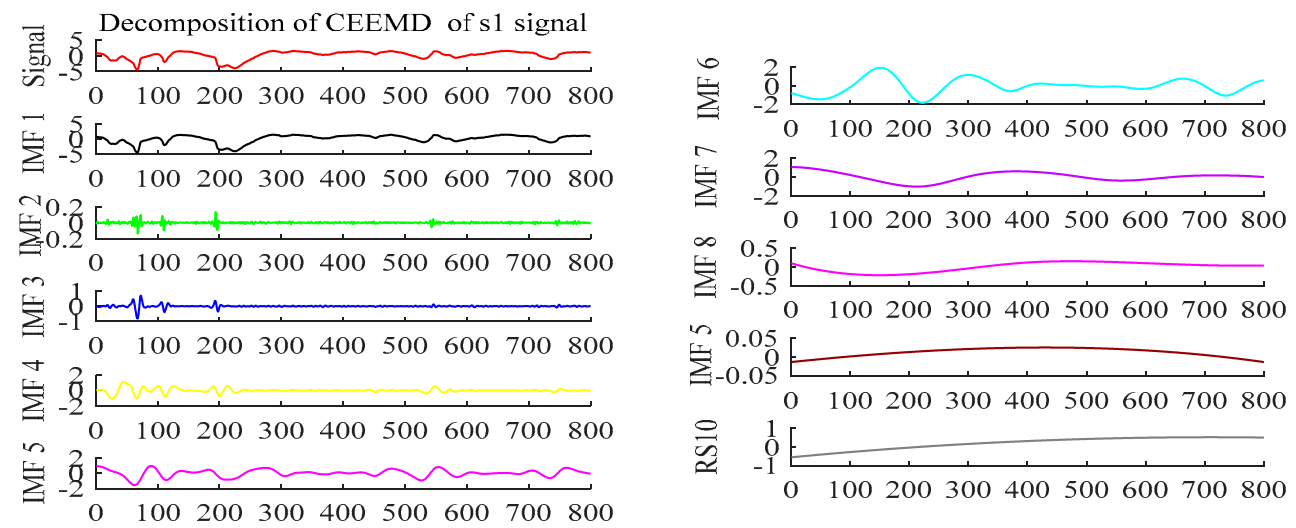

Figure 4. CEEMD decomposition IMF function and remainder.

In the test, CEEMD decomposition is performed for every specimen 300 column of two-dimensional signals. An $800 \times 8$ data matrix is composed of eight IMFs for singular value decomposition. The singular values are extracted and accumulated after each decomposition. Then, the skid-resistance index $\sigma_{\mathrm{i}}$ is obtained. Table 1 shows the regression analysis based on the data on the eight sets of testing areas. The linear fittings are obtained between the $\sigma_{\mathrm{i}}$ and the pendulum, measured by the British Pendulum Tester. The results show that the $R^{2}$ of $\sigma_{1}-\sigma_{5}$ and the friction coefficient is greater than 0.8 . The $\sigma_{6}-\sigma_{8}$ fitting is so poor that the $\mathrm{R}^{2}$ is less than 0.6. In Figure 5, the horizontal axis represents the third group of eight singular values in $\sigma_{3}$, and the vertical axis represents the measured values of BPN. The $\mathrm{R}^{2}$ of $\sigma_{3}$ and the pendulum value is up to 0.877 , and the fitting straight line is shown in Figure 5. The fitting Equation (6) is:

$$
\mathrm{y}=0.0078 \mathrm{x}+0.3439
$$

Table 1. $R^{2}$ goodness of fit.

\begin{tabular}{ccccccccccc}
\hline $\begin{array}{c}\text { Type } \\
(\mathrm{IMF}=8)\end{array}$ & $\boldsymbol{\sigma}_{1}$ & $\boldsymbol{\sigma}_{2}$ & $\boldsymbol{\sigma}_{3}$ & $\boldsymbol{\sigma}_{4}$ & $\boldsymbol{\sigma}_{5}$ & $\boldsymbol{\sigma}_{6}$ & $\boldsymbol{\sigma}_{7}$ & $\boldsymbol{\sigma}_{8}$ & $\sum_{\mathbf{i}=1}^{5} \boldsymbol{\sigma}_{\mathbf{i}}$ & $\sum_{\mathbf{i}=1}^{5} \boldsymbol{\sigma}_{\mathbf{i}}$ \\
\hline $\mathrm{R}^{2}(\mathrm{BPN})$ & 0.828 & 0.856 & 0.877 & 0.857 & 0.812 & 0.52 & 0.251 & 0.329 & 0.859 & 0.858 \\
\hline
\end{tabular}

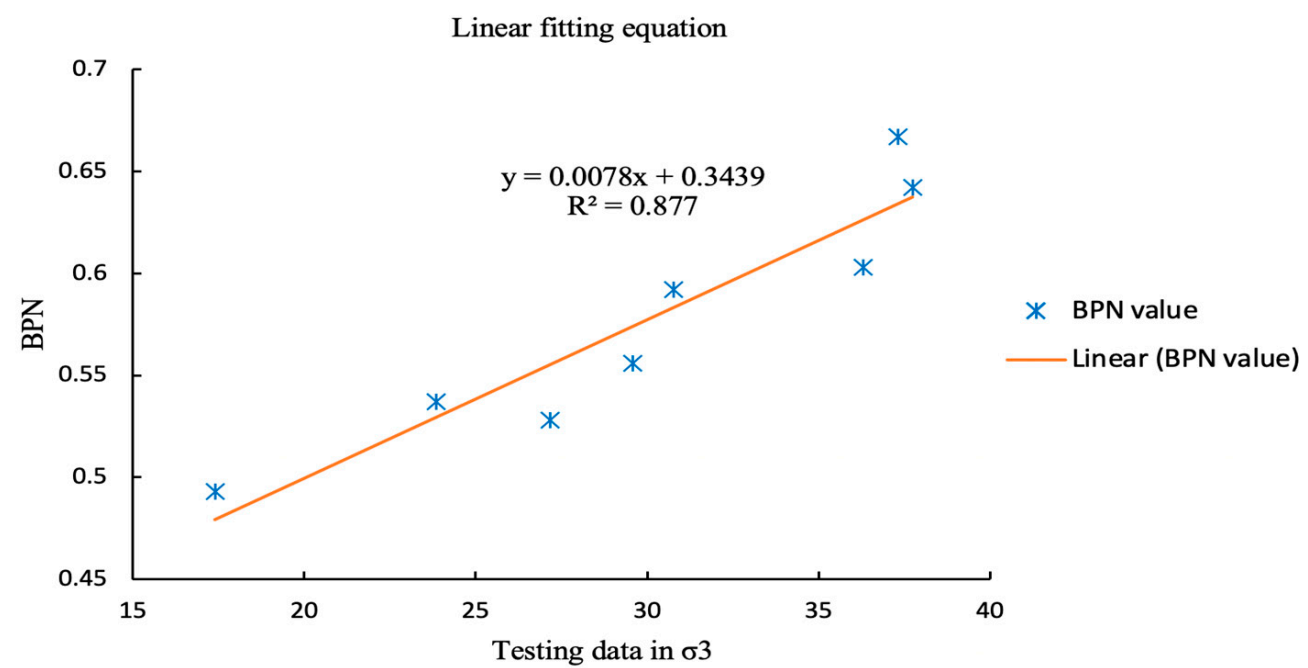

Figure 5. BPN and Testing data in $\sigma_{3}$ linear fitting equation. 
There is a certain correlation between $\sigma_{\mathrm{i}}(\mathrm{i} \leq 5)$ and the friction coefficient. When all the $\sigma_{i}$ are summed, there is also a good correlation between the summation of $\sum_{i=1}^{8} \sigma_{i}$ and the friction coefficient. Since the fit of $\sigma_{6}-\sigma_{8}$ is poor, we can accept that the correlation between the summation of $\sum_{i=1}^{5} \sigma_{i}$ and the friction coefficient is 0.859 , which is only 0.001 different from the sum of all $\sigma_{i}$. In terms of numerical magnitude, it is mainly because $\sigma_{i j}$ is arranged from large to small. The value of the $\sigma_{6}-\sigma_{8}$ is relatively small compared to the former. In terms of frequency, $\sigma_{6}-\sigma_{8}$ correspond to the IMFs' low frequency signal. Low frequency of the texture corresponds to the mega-texture, which has little impact on the skid-resistance performance of the pavement.

To further verify the reliability of the indicators, the contour arithmetic mean deviation $R_{a}$ is calculated. $R_{a}$ is the arithmetic means of the absolute value of contour deviation within the sampling range. From a statistical perspective, $R_{a}$ takes the amplitude distribution of the surface texture morphology of the road surface as its average. It also represents the absolute first-order origin distance of the set of data points, reflecting the dispersion degree of the contour amplitude of the topography relative to the reference line. It is widely used to evaluate the roughness of the surface morphology features. The $\mathrm{R}_{\mathrm{a}}$ is calculated using Equation (7), with the use of 3D texture data.

$$
\mathrm{R}_{\mathrm{a}}=\frac{1}{\mathrm{M} \times \mathrm{N}} \sum_{\mathrm{i}=1}^{\mathrm{N}} \sum_{\mathrm{j}=1}^{\mathrm{M}}\left|\mathrm{Z}\left(\mathrm{x}_{\mathrm{i}}-\mathrm{y}_{\mathrm{i}}\right)\right|
$$

where $\mathrm{Z}(\mathrm{x}, \mathrm{y})$ is the elevation information of topography based on datum line; $\mathrm{M}$ and $\mathrm{N}$ are the sampling points in two perpendicular directions.

In Table 2, the correlation of $\sigma_{i}$ and BPN with $R_{a}$ were calculated. It can be seen that $\sigma_{1}$ to $\sigma_{5}$ have a good correlation with $R_{a}$ while $\sigma_{6}$ to $\sigma_{8}$ have a poor correlation. Finally, the goodness of fit of $R_{a}$ and BPN is 0.772 . The best correlation in Table 2 is $\sigma_{1}$ where $R^{2}$ is up to 0.944 . In Figure 6 , the linear fitting equations of $\sigma_{1}$ and BPN with $R_{a}$ were calculated. $R_{a}$ as a widely used index of texture evaluation has a strong correlation with $\sigma_{i}$. Thus, it proves that $\sigma_{i}$ can well-reflect the skid-resistance performance of the pavement surface.

Table 2. The correlation of $\sigma_{\mathrm{i}}$ and BPN with $\mathrm{R}_{\mathrm{a}}$.

\begin{tabular}{cccccccccccc}
\hline $\begin{array}{c}\text { Type } \\
(\mathrm{IMF}=8)\end{array}$ & $\boldsymbol{\sigma}_{1}$ & $\boldsymbol{\sigma}_{2}$ & $\boldsymbol{\sigma}_{3}$ & $\boldsymbol{\sigma}_{4}$ & $\boldsymbol{\sigma}_{5}$ & $\boldsymbol{\sigma}_{6}$ & $\boldsymbol{\sigma}_{7}$ & $\boldsymbol{\sigma}_{8}$ & $\sum_{\mathbf{i}=1}^{5} \boldsymbol{\sigma}_{\mathbf{i}}$ & $\sum_{\mathbf{i}=1}^{5} \boldsymbol{\sigma}_{\mathbf{i}}$ & $\mathbf{B P N}$ \\
\hline $\mathrm{R}^{2}\left(\mathrm{R}_{\mathrm{a}}\right)$ & 0.944 & 0.922 & 0.919 & 0.901 & 0.905 & 0.529 & 0.358 & 0.377 & 0.941 & 0.935 & 0.772 \\
\hline
\end{tabular}

At first, the number of decompositions by CEEMD is set at eight. When the decomposition of the numbers is more than eight, the changing trend and the shape of IMF9 and IMF10 are consistent, and only the decomposition frequencies are reduced. When the number of decompositions is less than eight, the decomposition of the original signal is not sufficient, and the feature information of the original signal cannot be fully extracted. When the decomposition number of the IMF is changed-that is, when the number of IMF decomposition is greater than eight-there is a good correlation between the number of IMF and the friction value from IMF2 to IMF6. Evidently, they are the same as the one with IMF equal to eight.

When the number of IMF decomposition is greater than eight, the correlation of $\sigma_{x}$ $(\mathrm{x} \leq \mathrm{i}-2)$ and the pendulum value is greater than 0.8 . This shows that there is a good correlation between the number of IMF and the friction value from IMF2 to IMF6. When the number of IMF decomposition is less than eight, the $\sigma_{i}$ numbers that $\mathrm{R}^{2}$ more than 0.8 gradually decrease (Figure 7). Therefore, when the number of CEEMD decomposition is set at eight, texture signals can be effectively decomposed and each modal signal can be fully decomposed. 


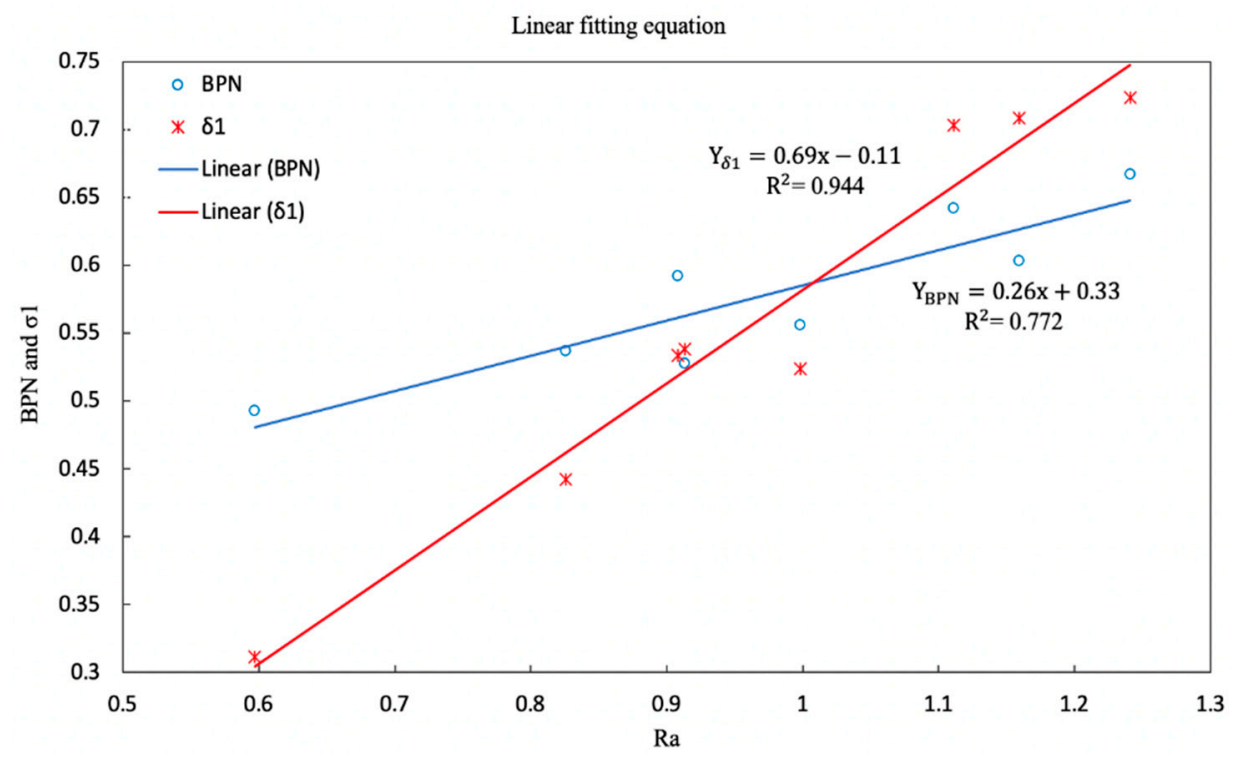

Figure 6. BPN and $\sigma_{i}$ with $\mathrm{R}_{\mathrm{a}}$ linear fitting equation.

When the IMF number is set to be less than eight, the last two $\sigma_{x}(i-2<x \leq i$, where $i$ is the IMF number) for each decomposition will have a poor fit with the pendulum value.

The number of $\mathrm{R}^{2}$ more than 0.8

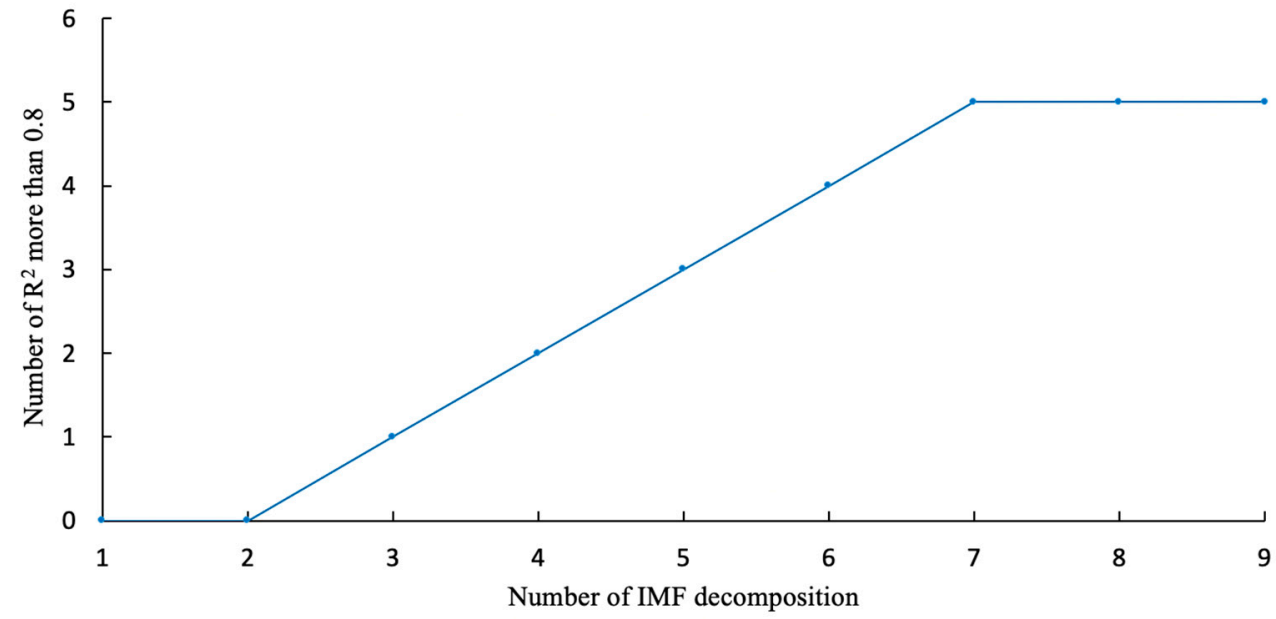

Figure 7. The number of $\mathrm{R}^{2}$ more than 0.8 vs. number of IMF decomposition.

In summary, it is evident that texture signal is featured as a complex signal. The texture information collected by the sensor is the direct information obtained from the spatial domain. In this study, the original texture signals are decomposed by CEEMD and SVD. Complex signals are decomposed into single modal functions and are representative of eigenvectors. By summation of the singular value, the texture skid-resistance parameter $\sigma_{i}$ is obtained. It is found that $\sigma_{i}$ is well correlated with the pavement friction value. The underlying non-contact texture testing module collects abundant 3D pavement texture information. By analyzing texture information, the skid-resistance performance index can be obtained quickly by calculating $\sigma_{i}$.

\section{Conclusions}

This study presents a non-contact texture data collection device based on online scanning laser sensor technology. The 3D pavement texture information is obtained by the device. In-depth research was conducted with a focus on the texture of the HMA surface. It mainly includes the following data analysis process: (1) obtain 2D and 3D 
texture information of HMA specimens by 3D laser constructor and decompose the 2D original signal by CEEMD algorithm to obtain $800 \times 10$ data matrix, and (2) remove IMF1 and RS10, where the eight value vectors $\sigma_{\mathrm{ij}}$ are obtained by performing singular value decomposition on $800 \times 8$ data matrix and the skid-resistance performance parameter $\sigma_{i}$ is obtained.

The key findings are that (1) when the number of IMF decomposition is greater than eight, there is a good correlation between the number of IMF and the friction value of texture from IMF2 to IMF6, and (2) when the number of IMF decomposition is less than eight, the $\sigma_{i}$ numbers that $R^{2}$ more than 0.8 gradually decrease.

With the increasing requirements in road maintenance and management, rapid detection and evaluation technology is becoming more and more important. Through the correlation analysis between $\sigma_{\mathrm{i}}$ and the friction coefficient, it is shown that this approach can provide data support for the transportation infrastructure management platform quickly and efficiently. It will also expectantly benefit decision-making network-level pavement management.

Author Contributions: Conceptualization, T.F. and R.G.; methodology, T.F.; formal analysis, R.G. and Y.S.; investigation, Y.S. and T.F.; resources, T.F.; data analysis, R.G. and T.F.; writing-original draft preparation, T.F., R.G. and Y.S.; writing - review and editing, T.F. and Y.S.; visualization, T.F. and Y.S.; supervision, R.G.; project administration, T.F. All authors have read and agreed to the published version of the manuscript.

Funding: This research received no external funding.

Institutional Review Board Statement: Not applicable.

Informed Consent Statement: Not applicable.

Data Availability Statement: Restrictions apply to the availability of these data. The data presented in this study are available on request from the corresponding author.

Acknowledgments: The authors wish to thank all the technicians, teachers, and students who contributed and shared in this study. Also, the authors are grateful to the journal editor and the anonymous reviewers for their helpful comments and suggestions.

Conflicts of Interest: The authors declare no conflict of interest.

\section{References}

1. Pei, Y.L. Road Traffic Safety; China Communication Press: Beijing, China, 2004.

2. Liu, Y.; Fwa, T.F.; Choo, Y.S. Finite-element modeling of skid resistance test. J. Transp. Eng. 2003, 129, 316-321. [CrossRef]

3. Zahouani, H.; Vargiolu, R.; Do, M.T. Characterization of microtexture related to wet road/tire friction. In Proceedings of the SURF 2000: Fourth International Symposium on Pavement Surface Characteristics on Roads and Airfields World Road Association (PIARC), Nantes, France, 22-24 May 2000.

4. Holzwarth, F.; Eichhorn, U. Non-contact sensors for road conditions. Sens. Actuators A Phys. 1993, 37, 121-127. [CrossRef]

5. Gong, F.; Yao, S.; Liu, Y.; You, Z.; Wang, H. A new method for characterizing coarse aggregate morphology through a MATLAB program. Geotech Sp. 2016, 262, 53-60.

6. Li, L.; Wang, K.C.; Li, Q. Geometric texture indicators for safety on AC pavements with $1 \mathrm{~mm} 3 \mathrm{D}$ laser texture data. Int. J. Pavement Res. Technol. 2016, 9, 49-62. [CrossRef]

7. Vlădescu, S.-C.; Medina, S.; Olver, A.V.; Pegg, I.G.; Reddyhoff, T. The transient friction response of a laser-textured, reciprocating contact to the entrainment of individual pockets. Tribol. Lett. 2016, 62, 19. [CrossRef]

8. Chou, C.-P.; Lee, C.-C.; Chen, A.-C.; Wu, C.-Y. Using a constructive pavement texture index for skid resistance screening. Int. J. Pavement Res. Technol. 2017, 10, 360-368. [CrossRef]

9. Meegoda, J.N.; Gao, S. Evaluation of pavement skid resistance using high speed texture measurement. J. Traffic Transp. Eng. 2015, 2, 382-390. [CrossRef]

10. Zhou, X.L.; Zhu, Y.Y.; Ran, M.P.; Xiao, S.Q.; Huang, X.M. Analysis of polishing behavior of asphalt pavement surface based on segmenting variable dimension. China J. Highw. Transp. 2019, 32, 187-195.

11. Yang, G.F.; Wang, H.Y.; Pan, Y.L. Wear detection and evaluation method based on multi-line texture. China J. Highw. Transp. 2016, $29,35-40$.

12. Rado, Z.; Kane, M. An initial attempt to develop an empirical relation between texture and pavement friction using the HHT approach. Wear 2014, 309, 233-246. [CrossRef] 
13. Choongwoo, C. Application of Hilbert Huang Transformation to Analyze Pavement Texture-Friction Relationship. Ph.D. Thesis, The Pennsylvania State University, State College, PA, USA, 2010.

14. Huang, N.E.; Shen, Z.; Long, S.R.; Wu, M.C.; Shih, H.H.; Zheng, Q.; Yen, N.-C.; Tung, C.C.; Liu, H.H. The empirical mode decomposition and Hilbert spectrum for non-linear and non-stationary time series analysis. Proc. R. Soc. Lond. A. 1998, 454, 903-995. [CrossRef]

15. Huang, N.E.; Shen, Z.; Long, S.R. A new view of nonlinear water waves: The Hilbert spectrum. Annu. Rev. Fluid Mech. 1999, 31, 417-457. [CrossRef]

16. $\mathrm{Wu}, \mathrm{Z}$; Huang, N.E. Ensemble empirical mode decomposition: A noise-assisted data analysis method. Adv. Adapt. Data Anal. 2009, 1, 1-41. [CrossRef]

17. Yeh, J.-R.; Shieh, J.-S.; Huang, N.E. Complementary ensemble empirical mode decomposition: A novel noise enhanced data analysis method. Adv. Adapt. Data Anal. 2010, 2, 135-156. [CrossRef]

18. Si, Y.Q.; Guo, R.H.; Shi, P.C. Comparative study of signal time-frequency analysis techniques based on EMD, EEMD and CEEMD. CT Theory Appl. 2019, 28, 417-426. (In Chinese) 